\title{
A GENERAL MEASURE DECOMPOSITION THEOREM BY MEANS OF THE GENERALIZED WALLMAN REMAINDER
}

\author{
GEORGE BACHMAN and PANAGIOTIS D. STRATIGOS
}

(Received 5 April 1982)

Communicated by W. Moran

\begin{abstract}

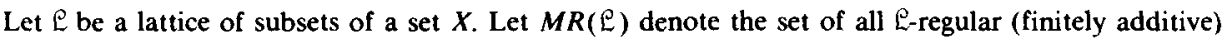
measures on the algebra generated by $\mathcal{L}$. Under the assumption that $\mathcal{E}$ is disjunctive, in the first part of the paper, criteria are obtained for the $\sigma$-smoothness, $\tau$-smoothness, and tightness of elements of $M R(E)$ in terms of the general Wallman remainder. In the second part of the paper, various applications are given, and, in particular, extensions and refinements of the Yosida-Hewitt Decomposition Theorem are obtained.
\end{abstract}

1980 Mathematics subject classification (Amer. Math. Soc.): 28 A 60, 28 A 32.

\section{Introduction}

Let $\mathcal{E}$ be a lattice of subsets of $X$, and $\mathfrak{A}(\mathcal{E})$ the algebra generated by $\mathcal{L} . M R(\mathcal{C})$

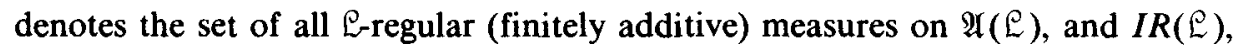
the set of all $0-1$ valued measures in $M R(\varrho)$. The topological space $\langle I R(\mathfrak{E}), t W(\mathfrak{E})\rangle$, where $W(\mathfrak{L})$ is the Wallman lattice, is the general Wallman space.

In the first part of this paper, we show that, in case $\mathcal{L}$ is disjunctive, each $\mu \in M R(\mathcal{E})$ induces two measures $\hat{\mu}$ and $\tilde{\mu}$, defined on certain algebras of subsets of $I R(\mathcal{L})$. Then, we give criteria for $\sigma$-smoothness, $\tau$-smoothness, and tightness in terms of $\hat{\mu}$ or $\tilde{\mu}$, and the general Wallman remainder $I R(e)-X$.

(C) 1984 Australian Mathematical Society $0263-6115 / 84 \$ A 2.00+0.00$ 
These results greatly generalize earlier work, [4], where it was necessary to assume that $\mathcal{L}$ was also $\delta$ and normal in order that the Alexandroff Representation Theorem, [1], could be applied to induce the measures $\hat{\mu}$ and $\tilde{\mu}$. This approach is now bypassed by using general measure extension theorems. We note that this earlier work had already generalized the work of Knowles, [10], and Gould and Mahowald, [8], in which $X$ was a Tychonoff space and $\mathscr{L}$ the lattice of its zero sets.

We can now systematically apply our general criteria to important lattices which may not be $\delta$ or normal, such as the lattice of clopen sets in a $T_{2}$, 0-dimensional space, or the lattice of closed sets in a $T_{1}$ topological space, and for these special cases the results can be expressed in terms of subsets of $\beta_{0} X-X$, where $\beta_{0} X$ is the Banaschewski compactification of $X$, [6], and $\omega X-X$, where $\omega X$ is the Wallman compactification of $X$, [17], respectively. Thus, beside the well-known case of $\beta X-X$, we can develop, by this abstract approach, a unified treatment of certain aspects of topological measure theory, in terms of $I R(E)-X$, which will include all the important topological lattices.

However, beyond this, the general approach leads to new measure extension theorems, as well as to new criteria for lattice repleteness.

In the second part of the paper, various special measures are introduced, such as purely finitely additive, purely $\sigma$-additive, purely $\tau$-additive, strongly $\sigma$-additive, and so on and, again, utilizing the induced measures $\hat{\mu}$ and $\tilde{\mu}$ and certain subsets of the general Wallman space, we obtain various decomposition theorems which are extensions and refinements of the well-known Yosida-Hewitt Decomposition Theorem, [18].

\section{Terminology and notation}

Let $\mathcal{L}$ be any lattice of subsets of a set $X$. We shall assume, without loss of

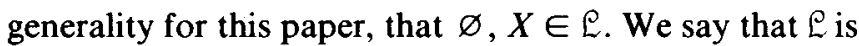

1) $\delta$ if $\mathcal{L}$ is closed under countable intersections;

2) complemented if for every $L \in \mathfrak{E}, L^{\prime} \in \mathfrak{E}$;

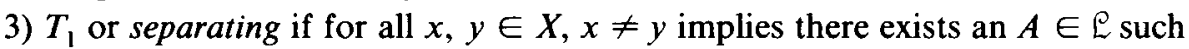
that $x \in A$ and $y \notin A$;

4) $T_{2}$ if for all $x, y \in X, x \neq y$ implies there exist $A, B \in \mathcal{E}$ such that $x \in A^{\prime}$, $y \in B^{\prime}$, and $A^{\prime} \cap B^{\prime}=\varnothing$;

5) disjunctive if for every $x \in X$ and every $A \in \mathcal{L}, x \notin A$ implies there exists a $B \in \mathcal{E}$ such that $x \in B$ and $A \cap B=\varnothing$; 
6) regular if for every $x \in X$ and every $A \in \mathcal{L}, x \notin A$ implies there exist $B$, $C \in \mathcal{L}$ such that $x \in B^{\prime}, A \subset C^{\prime}$, and $B^{\prime} \cap C^{\prime}=\varnothing$;

7) normal if for all $A, B \in \mathcal{E}, A \cap B=\varnothing$ implies there exist $C, D \in \mathcal{E}$ such that $A \subset C^{\prime}, B \subset D^{\prime}$, and $C^{\prime} \cap D^{\prime}=\varnothing$;

8) Lindelöf if any family of sets in $\mathcal{L}$ with the countable intersection property has a non-empty intersection;

9) compact if any family of sets in $\mathcal{E}$ with the finite intersection property has a non-empty intersection.

Similarly, one defines $\mathcal{E}$ is countably compact. $\mathcal{E}$ is countably paracompact if for any decreasing sequence $\left\langle A_{n}\right\rangle$ in $\varrho$ with $\lim _{n} A_{n}=\varnothing$, there exists a decreasing sequence $\left\langle B_{n}^{\prime}\right\rangle$ in $\mathcal{L}^{\prime}$ with $\lim _{n} B_{n}^{\prime}=\varnothing$ and $A_{n} \subset B^{\prime}$.

Next, let $\mathcal{E}_{1}$ and $\mathcal{E}_{2}$ be any lattices of subsets of $X$. $\mathcal{L}_{1}$ separates $\mathcal{L}_{2}$ if for all $A, B \in \mathfrak{E}_{2}, A \cap B=\varnothing$ implies there exist $C, D \in \mathfrak{L}_{1}$ such that $A \subset C, B \subset D$, and $C \cap D=\varnothing$.

$N$ designates the set of natural numbers. $\delta \mathcal{E}$ is the $\delta$ lattice generated by $\mathcal{E}$, and $t \mathscr{R}$ the lattice of arbitrary intersections of $E$-sets. A function from $X$ to $R \cup\{ \pm \infty\}$ is $\mathcal{L}$-continuous if the inverse image of every closed set is in $\mathcal{L}$. The set of $\mathcal{L}$-continuous functions is denoted by $C(\mathcal{E})$, and the set of bounded $\mathscr{E}$-continuous

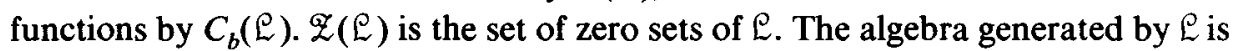
$\mathfrak{A}(\mathfrak{L})$, and the generated $\sigma$-algebra is $\sigma(\mathscr{E})$.

$M(\mathscr{E})$ denotes the set of all measures on $\mathfrak{A}(\mathscr{L})$; that is, those real-valued, bounded, finitely additive set functions on $\mathfrak{A}(\mathscr{Q})$. (See [1], page 567.) An element $\mu \in M(\mathfrak{e})$ is called:

1) R-regular if for each $A \in A(\mathscr{E})$ and every $\varepsilon>0$, there exists an $L \in \mathcal{L}$ such that $L \subset A$ and $|\mu(A)-\mu(L)|<\varepsilon$;

2) $\mathcal{L}-(\sigma$-smooth $)$ if for every decreasing sequence $\left\langle A_{n}\right\rangle$ in $\mathfrak{A}(\mathfrak{Q})$ with $\lim _{n} A_{n}=$ $\varnothing, \lim _{n} \mu\left(A_{n}\right)=0$ (see [16]);

3) $\mathcal{L}-(\tau$-smooth $)$ if for every decreasing net $\left\langle L_{\alpha}\right\rangle$ in $\mathcal{L}$ with $\lim _{\alpha} L_{\alpha}=\varnothing$, $\lim _{\alpha} \mu\left(L_{\alpha}\right)=0$ (see [16]);

4) $\mathcal{L}$-tight if $\mu \geqslant 0, \mu$ is $\mathcal{L}(\sigma$-smooth $)$, and for every $\varepsilon>0$, there exists an $\mathcal{E}$-compact set $K$ such that $\mu_{*}\left(K^{\prime}\right)<\varepsilon$ (see [10]).

$M R(\mathcal{L})$ is the subset of $M(\mathscr{L})$ of all $\mathcal{L}$-regular measures. $M(\sigma, \mathcal{E})$ is the subset of $M(\mathscr{L})$ of all $\mathcal{L}-(\sigma$-smooth) measures; while $M(\tau, \mathscr{L})$ is the set of all $\mathscr{L}-(\tau$-smooth $)$ measures, and $M(t, \mathcal{L})$ the set of all $\varrho_{-}$tight ones.

For $\mu \in M(\mathcal{E})$ with $\mu \geqslant 0$, the support of $\mu$ is $S(\mu)=\cap\{L \in \mathcal{L}\{\mu(L)=\mu(X)\}$.

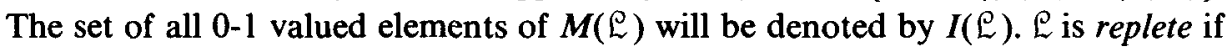
for every $\mu \in I R(\sigma, \mathcal{L}), S(\mu) \neq \varnothing$.

Since every element of $M(\mathscr{L})$ is the difference of nonnegative measures, in the sequel we shall work exclusively with nonnegative measures. 
In this section we work with an arbitrary set $X$ and a fairly arbitrary lattice $\mathscr{L}$ of subsets of $X$; with this pair we associate the general Wallman space $I R(\mathcal{L})$ (see below), and, for the general element of $M R(\mathcal{E})$, we investigate how the properties of $\sigma$-smoothness, $\tau$-smoothness, and tightness reflect over to $I R(\varrho)$ and conversely. The resulting theorems are not only intrinsically interesting, but they are also useful throughout the present paper.

2.0. Preliminaries. (i). Let $E$ be any lattice of subsets of $X$ such that $E$ is

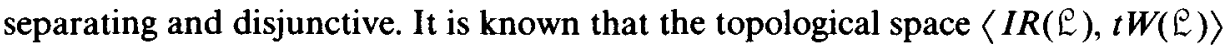
is compact and $T_{1}$; it is $T_{2}$ if and only if $\mathcal{E}$ is normal. (See, for example, [2] and [13].) Consider the map $\phi: X \rightarrow I R(\mathcal{E})$, where $\phi(X)=\mu_{x}$, the measure concentrated at $x . \phi$ is a $\langle t \mathfrak{E}, t W(\mathfrak{E}) \cap \phi(X)=t \phi(\mathfrak{E})\rangle$-homeomorphism. For this reason, $\phi(X)$ is topologically identifiable with $X$. Moreover, $\phi(X)$ is dense in $I R(\varrho)$. Consequently, $I R(\ell)$ is a compactification of $X$ if $X$ carries the $t 巴$ topology; it is known as the general Wallman compactification of $X$. In case $\phi(X)$ is identified with $X$, we say that $X$ is embedded in $I R(\ell)$.

For $A \in A(\mathcal{E})$, let $W(A)=\{\mu \in \operatorname{IR}(\mathcal{E}) \mid \mu(A)=1\}$, and $W_{\sigma}(A)=\{\mu \in$ $\operatorname{IR}(\sigma, \mathcal{E}) \mid \mu(A)=1\}$. The following statements are true:

1. If $A \in \mathfrak{A}(\mathcal{E})$, then $W(A)^{\prime}=W\left(A^{\prime}\right)$.

2. If $A, B \in \mathfrak{A}(\mathcal{E})$, then $\alpha) W(A \cup B)=W(A) \cup W(B) ; \beta) W(A \cap B)=$ $W(A) \cap W(B) ; \gamma) A \supset B$, if and only if $W(A) \supset W(B) ; \delta) A=B$, if and only if $W(A)=W(B)$.

3. $\mathfrak{A}(W(\mathfrak{E}))=W(\mathfrak{A}(\mathfrak{L}))$.

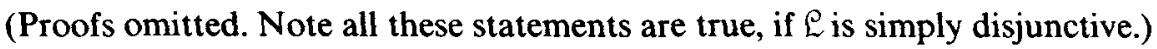

Next, let $\mu \in M(\mathfrak{L})$, and define $\hat{\mu}$ on $\mathfrak{A}(W(\mathfrak{E}))=W(\mathfrak{A}(\mathfrak{E}))$, by $\hat{\mu}(W(A))=$ $\mu(A)$, where $A \in \mathfrak{A}(\mathcal{E})$. Then, $\hat{\mu} \in M(W(\mathscr{L}))$ and, if $\mu \in M R(\mathcal{E})$, then $\hat{\mu} \in$ $M R(W(\mathfrak{E}))$. Conversely, let $\nu \in M(W(\mathfrak{E}))$, and define $\mu$ on $\mathfrak{A}(\mathfrak{E})$ by $\mu(A)=$ $\nu(W(A))$. Then, $\mu \in M(\mathfrak{E}), \nu=\hat{\mu}$, and if $\nu \in M R(W(\mathfrak{E}))$, then $\mu \in M R(\mathfrak{L})$.

Note, since $W(\mathscr{Q})$ is compact,

$$
M R(W(\varrho))=M R(\sigma, W(\varrho))=M R(\tau, W(\varrho))=M R(t, W(\varrho)) .
$$

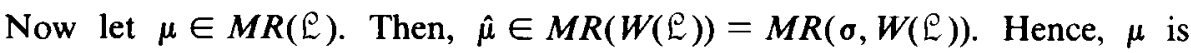
uniquely extendible to the $\sigma$-algebra of $\hat{\mu}^{*}$-measurable sets and the extension is $\delta W(\mathcal{E})$-regular. We will continue to use $\mu$ for this extension.

We note that $\mathfrak{A}\left(W_{\sigma}(\mathfrak{Q})\right)=W_{\sigma}(\mathfrak{A}(\mathfrak{Q}))$ (proof omitted). Now, let $\mu \in M(\mathscr{L})$ and define $\mu^{\prime}$ on $\mathfrak{A}\left(W_{\sigma}(\mathscr{L})\right)$ by $\mu^{\prime}\left(W_{\sigma}(B)\right)=\mu(B)$, where $B \in \mathfrak{A}(\mathcal{E})$. Then $\mu^{\prime} \in$ $M\left(W_{\sigma}(\mathcal{E})\right)$ and, if $\mu \in M R(\mathcal{E})$, then $\mu^{\prime} \in M R\left(W_{\sigma}(\mathcal{E})\right)$. Conversely, let $\rho \in$ $M\left(W_{\mathrm{o}}(\mathfrak{E})\right)$, and define $\mu$ on $\mathfrak{A}(\mathcal{L})$, by $\mu(B)=\rho\left(W_{\mathrm{o}}(B)\right)$. Then, $\mu \in M(\mathfrak{L}), \rho=\mu^{\prime}$, 
and, if $\rho \in M R\left(W_{\sigma}(\mathcal{L})\right)$, then $\mu \in M R(\varrho)$. The following statement is also true: If

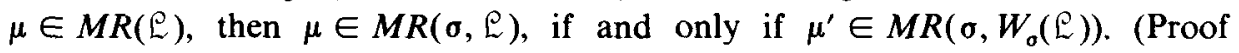
omitted.)

\subsection{On $\sigma$-smoothness.}

THEOREM 2.1. Let $\mathcal{L}$ be any lattice of subsets of $X$ such that $\mathcal{L}$ is (separating) and disjunctive. If $\mu \in M R(\mathcal{L})$, then the following statements are equivalent:

1. $\mu \in M R(\sigma, \mathcal{L})$.

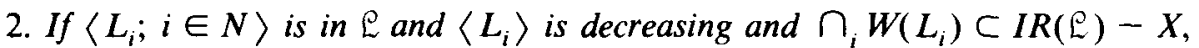
then $\hat{\mu}\left(\cap_{i} W\left(L_{i}\right)\right)=0$.

3. If $\left\langle L_{i} ; i \in N\right\rangle$ is in $\varrho$ and $\left\langle L_{i}\right\rangle$ is decreasing and $\cap_{i} W\left(L_{i}\right) \subset \operatorname{IR}(\varrho)-$ $\operatorname{IR}(\sigma, \mathcal{E})$, then $\hat{\mu}\left(\cap_{i} W\left(L_{i}\right)\right)=0$.

4. $\hat{\mu}^{*}(X)=\hat{\mu}(\operatorname{IR}(\mathcal{Q}))$.

5. $\hat{\mu}^{*}(\operatorname{IR}(\sigma, \mathcal{Q}))=\hat{\mu}(\operatorname{IR}(\mathcal{Q}))$.

Proof. $\alpha$ ). Assume 1 and let $\left\langle L_{i}\right\rangle$ be a decreasing sequence in $\varrho$ with $\cap_{i} W\left(L_{i}\right) \subset I R(\mathcal{L})-X$. We have $\hat{\mu}\left(\cap_{i} W\left(L_{i}\right)\right)=\lim _{i} \hat{\mu}\left(W\left(L_{i}\right)\right)=\lim _{i} \mu\left(L_{i}\right)$ $=\mu\left(\cap_{i} L_{i}\right)=0$ since $\cap_{i} L_{i}=\varnothing$. Hence, 2 follows. Conversely, assume 2 and let $\left\langle L_{i}\right\rangle$ be a decreasing sequence in $\mathcal{E}$ with $\lim _{i} L_{i}=\varnothing$. Then, $\cap_{i} W\left(L_{i}\right) \subset$ $I R(\mathfrak{E})-X$, for, if not, there exists an $x \in X$ with $\mu_{x}\left(L_{i}\right)=1$ for all $i$, which is absurd. It now follows that $\mu \in M R(\sigma, Q)$.

$\beta)$. The proof that 1 and 3 are equivalent is similar and will be omitted.

$\gamma)$. Since $\hat{\mu}^{*}(X)+\hat{\mu}_{*}(\operatorname{IR}(\mathcal{E})-X)=\hat{\mu}(\operatorname{IR}(\mathcal{E}))$ and $\hat{\mu}_{*}(\operatorname{IR}(\mathcal{E})-X)=$

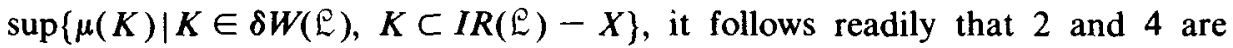
equivalent.

$\delta$ ). To show 3 and 5 are equivalent, the same type argument as in $\gamma$ ) is used.

REMARK. The part of the assumption " $E$ is separating" is not needed, in case $\phi(X)$ is not identified with $X$. Whenever we wish to indicate this in a theorem, we shall enclose the word "separating" in parentheses.

ObSERVATION. 4 and 5 are equivalent to

$4^{\prime} . X$ is $\hat{\mu}$-thick;

$5^{\prime} . \operatorname{IR}(\sigma, \mathcal{Q})$ is $\hat{\mu}$-thick

(see [9], pages 74, 75). 
2.2. On $\tau$-smoothness.

LEMMA 2.1. Let $\mathfrak{L}_{1}$ and $\mathfrak{L}_{2}$ be any lattices of subsets of $X$ such that $\mathfrak{L}_{1} \subset \mathfrak{L}_{2}$. If $\mu \in M R\left(\mathcal{L}_{1}\right)$, then $\mu$ can be extended to a $\nu \in M R\left(\mathfrak{L}_{2}\right)$, and, if $\mathfrak{L}_{1}$ separates $\mathfrak{L}_{2}$, the extension is unique. (See [5] and [11].)

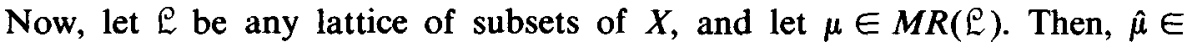
$M R(W(\varrho))$, and, therefore, by the lemma, $\hat{\mu}$ can be extended to a $\tilde{\mu} \in M R(t W(\mathscr{E}))$. Moreover, since $W(\mathfrak{E})$ is compact, $W(\mathfrak{E})$ separates $t W(\mathscr{E})$, and, therefore, the extension is unique. Also, since $M R(t W(\mathfrak{E}))=M R(\sigma, t W(\mathfrak{E})), \tilde{\mu}$ is uniquely extendible to the $\sigma$-algebra of $\tilde{\mu}^{*}$-measurable sets and the extension is also $t W(\mathcal{L})$-regular. We continue to use $\hat{\mu}$ for this extension.

LEMMA 2.2. Let $\mathcal{L}$ be any lattice of subsets of $X$ which is $\delta$. The following statements are equivalent:

1. $\mu \in M R(\tau, \varrho)$.

2. If $\left\langle L_{\alpha} ; \alpha \in A\right\rangle$ (net) is in $E$ and $\left\langle L_{\alpha}\right\rangle$ is decreasing, then $\mu^{*}\left(\cap_{\alpha} L_{\alpha}\right)=$ $\inf _{\alpha} \mu\left(L_{\alpha}\right)$.

3. If $\left\{L_{\alpha} ; \alpha \in A\right\} \subset \mathcal{Q}$ and $\left\{L_{\alpha} ; \alpha \in A\right\}$ is a filter base, then $\mu^{*}\left(\cap_{\alpha} L_{\alpha}\right)=$ $\inf _{\alpha} \mu\left(L_{\alpha}\right)$. (See [14].)

We can now establish, quite analogously to the proof of Theorem 2.1 , the following:

THEOREM 2.2. Let $\mathrm{E}$ be any lattice of subsets of $X$ such that $\mathcal{L}$ is (separating) and

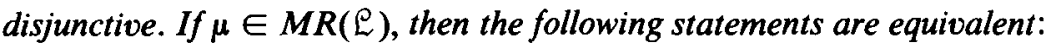

1. $\mu \in M R(\tau, \mathscr{L})$.

2. If $\left\langle L_{\alpha} ; \alpha \in A\right\rangle$ (net) is in $\mathcal{E}$ and $\left\langle L_{\alpha}\right\rangle$ is decreasing and $\cap_{\alpha} W\left(L_{\alpha}\right) \subset \operatorname{IR}(\mathcal{L})$ $-X$, then $\tilde{\mu}\left(\cap_{\alpha} W\left(L_{\alpha}\right)\right)=0$.

3. $\tilde{\mu}^{*}(X)=\tilde{\mu}(I R(\mathcal{L}))$.

ObSERvation. Statement 2 is equivalent to the statement: If $K \in t W(\mathfrak{L})$ and $K \subset I R(\mathcal{E})-X$, then $\tilde{\mu}(K)=0$.

THEOREM 2.3. Let $\mathcal{E}$ be any lattice of subsets of $X$ such that $\mathcal{L}$ is separating and disjunctive. If $\mu \in M R(\tau, \mathcal{L})$, then $\mu$ can be extended to a $\nu \in M R(\tau, t \mathfrak{L})$ such that

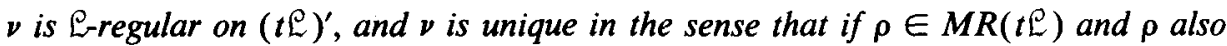
extends $\mu$, then $\rho=\nu$. 
Proof. (i). Existence. Since $\mu \in M R(\tau, \mathfrak{L})$, by Theorem 2.2, $\tilde{\mu}^{*}(X)=\tilde{\mu}(\operatorname{IR}(\mathfrak{L}))$; that is, $X$ is $\tilde{\mu}$-thick, and the projection of $\tilde{\mu}$ on $A(t \mathfrak{L})$, denoted by $\nu$, is defined. Then, for $A \in A(t \mathfrak{L})$, there exists an $A^{*} \in \mathfrak{A}(t W(\mathfrak{L}))$ such that $A=A^{*} \cap X$ and $\nu(A)=\tilde{\mu}\left(A^{*}\right)$. It is readily seen that $\nu$ extends $\mu$, and $\nu \in M R(\tau, t \varrho)$.

(ii). Now, we show that $\nu$ is $\mathcal{G}$-regular on $(t \mathcal{L})^{\prime}$. Let $B \in(t \mathfrak{L})^{\prime}$, and $\varepsilon>0$. Then, $B=G \cap X$, where $G \in(t W(\mathfrak{L}))^{\prime}$. By the regularity of $\tilde{\mu}$, there exists a $K \in t W(\mathfrak{L})$ such that $K \subset G$ and $\tilde{\mu}(G-K)<\varepsilon$. However, $K=\bigcap_{\alpha} W\left(L_{\alpha}\right), L_{\alpha} \in \mathcal{L}$, so $\left(\cap_{\alpha} W\left(L_{\alpha}\right)\right) \cap G^{\prime}=\varnothing$, and, by compactness, there exist finitely many of the $L_{\alpha}$, say, $L_{\alpha_{1}}, \ldots, L_{\alpha_{n}}$ such that $\left(\cap_{i=1}^{n} W\left(L_{\alpha_{i}}\right)\right) \cap G^{\prime}=\varnothing$. Thus, $W(\tilde{L}) \cap G^{\prime}=\varnothing$, where $\tilde{L}=\cap_{i=1}^{n} L_{\alpha_{i}} \in \mathcal{L}$. Hence, $K \subset W(\tilde{L}) \subset G$, and $W(\tilde{L}) \cap X \subset G \cap X=$ $B$, so $\tilde{L} \subset B$. Finally, $\nu(B-\tilde{L})=\nu((G-W(\tilde{L})) \cap X)=\tilde{\mu}(G-W(\tilde{L})) \leqslant$ $\tilde{\mu}(G-K)<\varepsilon$, which completes (ii).

(iii) (We omit the proof of uniqueness.)

REMARK. For a related type of extension involving content see [15].

We will also need the following theorem, the proof of which is in the same spirit as that of Theorems 2.1 and 2.2, and will be omitted.

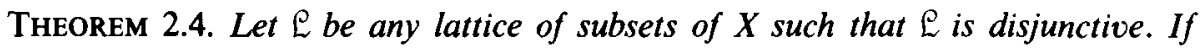
$\mu \in M R(\mathcal{E})$, then the following statements are equivalent:

1. $\mu^{\prime} \in M R\left(\tau, W_{0}(\mathcal{L})\right)$.

2. If $\left\langle W\left(L_{\alpha}\right) ; \alpha \in A\right\rangle$ (net) is in $\mathcal{E}$ and $\left\langle W\left(L_{\alpha}\right)\right\rangle$ is decreasing and $\cap_{\alpha} W\left(L_{\alpha}\right)$ $\subset \operatorname{IR}(\varrho)-\operatorname{IR}(\sigma, \varrho)$, then $\tilde{\mu}\left(\cap_{\alpha} W\left(L_{\alpha}\right)\right)=0$.

3. $\tilde{\mu}^{*}(\operatorname{IR}(\sigma, E))=\tilde{\mu}(\operatorname{IR}(\varrho))$.

OBSERVATION. Clearly, statement 2 is equivalent to the statement: If $K \in t W(\varrho)$

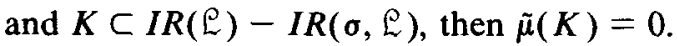

\subsection{On tightness.}

THEOREM 2.5. Let $\mathcal{E}$ be any lattice of subsets of $X$ such that $\mathcal{E}$ is separating, disjunctive, and normal. If $\mu \in M R(\mathcal{E})$, then the following statements are equivalent:

1. $\mu \in M R(t, \mathfrak{E})$.

2. $\tilde{\mu}^{*}(X)=\tilde{\mu}(\operatorname{IR}(\mathfrak{E}))$ and $X$ is $\tilde{\mu}^{*}$-measurable.

Proof. Assume 1 and let $\varepsilon>0$. Then, there exists an $\mathcal{L}$-compact set $K$ such that $\mu_{*}\left(K^{\prime}\right)<\varepsilon$. Since $\mu \in M R(t, \mathcal{L})$, it follows that $\mu \in M R(\tau, \mathfrak{L})$, and, therefore, by 
Theorem 2.3, there exists a unique $\nu \in M R(\tau, t \mathfrak{L})$ extending $\mu$ and $\mathcal{L}$-regular on $(t \mathscr{L})^{\prime}$. Also, by the hypothesis, it follows that $K \in t \mathscr{L}$, so $K^{\prime} \in(t \mathscr{L})^{\prime}$. Let $\mu$ be extended to $\sigma(\mathcal{L})$ and $\nu$ to $\sigma(t \mathfrak{L})$. Then,

$$
\nu\left(K^{\prime}\right)=\sup \left\{\nu(L) \mid L \in \mathcal{L}, L \subset K^{\prime}\right\}
$$

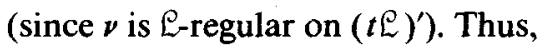

$$
\begin{aligned}
\nu\left(K^{\prime}\right) & =\sup \left\{\mu(L) \mid L \in \mathcal{L}, L \subset K^{\prime}\right\} \\
& \leqslant \sup \left\{\mu(E) \mid E \in \sigma(\mathfrak{L}), E \subset K^{\prime}\right\} \\
& =\sup \left\{\nu(E) \mid E \in \sigma(\mathfrak{L}), E \subset K^{\prime}\right\} \\
& \leqslant \sup \left\{\nu(E) \mid E \in \sigma(t \mathcal{L}), E \subset K^{\prime}\right\} \\
& =\nu\left(K^{\prime}\right) .
\end{aligned}
$$

Therefore, $\nu\left(K^{\prime}\right)=\sup \left\{\mu(E) \mid E \in \sigma(\mathscr{Q}), E \subset K^{\prime}\right\}=\mu_{*}\left(K^{\prime}\right)$. Also, $K^{\prime}=X-K$ $=(I R(\mathfrak{E})-K) \cap X$ and, since $K$ is $t W(\mathfrak{E})$-compact and $t W(\mathscr{E})$ is $T_{2}$ (by the normality of $\mathcal{E}), K \in t W(\mathfrak{L})$. Then,

$$
\begin{aligned}
\tilde{\mu}^{*}(I R(\mathcal{L})-X) & \leqslant \tilde{\mu}(I R(\mathcal{L})-K)=\nu((I R(\mathfrak{L})-K) \cap X) \\
& =\nu\left(K^{\prime}\right)=\mu_{*}\left(K^{\prime}\right)<\varepsilon .
\end{aligned}
$$

Consequently, $\tilde{\mu}^{*}(\operatorname{IR}(\mathcal{E})-X)=0$, and 2 follows.

Conversely, assume 2 and let $\varepsilon>0$. Since $X$ is $\tilde{\mu}^{*}$-measurable and $\tilde{\mu}$ is $t W(\mathfrak{L})$-regular on the $\sigma$-algebra of $\tilde{\mu}^{*}$-measurable sets, $\tilde{\mu}^{*}(X)=\sup \{\tilde{\mu}(K) \mid K \in$ $t W(\mathcal{E}), K \subset X\}$. Thus, there exists a $K \in t W(\varrho), K \subset X$ such that $\tilde{\mu}(K)>\tilde{\mu}^{*}(X)$ - $\varepsilon$. $K$ is, of course, $\mathcal{E}$-compact; also, $K \in t \mathfrak{L}$, and, as above, $\nu\left(K^{\prime}\right)=\mu_{*}\left(K^{\prime}\right)$, so $\nu(K)=\mu^{*}(K)$; but, $\nu(K)=\nu(K \cap X)=\tilde{\mu}(K)$. Hence, $\mu^{*}(K)>\tilde{\mu}^{*}(X)-\varepsilon=$ $\tilde{\mu}(\operatorname{IR}(\mathcal{L}))-\varepsilon=\mu(X)-\varepsilon$, which establishes 1 . Note, in this part the normality of $E$ is not needed.

In this section we introduce two important sets of measures and establish certain lemmas critical for the subsequent development.

Consider any disjunctive lattice $\mathcal{E}$ of subsets of $X$.

The set $M R^{\prime}(\mathcal{E}): \mu \in M R^{\prime}(\mathfrak{L})$ if $\mu \in \operatorname{MR}(\mathcal{L})$ and $\mu^{\prime} \in \operatorname{MR}\left(\tau, W_{\mathrm{o}}(\mathfrak{E})\right)$.

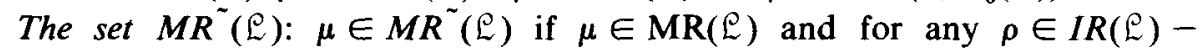
$I R(\sigma, \mathcal{L})$, there exists a $G \in(t W(\mathscr{L}))^{\prime}$ such that $\rho \in G$ and $\tilde{\mu}(G)=0$.

By a compactness argument, it clearly follows that $M R^{\tilde{N}}(\mathcal{E}) \subset M R^{\wedge}(\mathcal{E})$.

The following two theorems indicate the importance of these sets concerning repleteness. 
THEOREM 3.1. (On $M R^{\wedge}(\mathcal{Q})$.) The following statements are true:

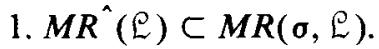

2. $I R(\sigma, E) \subset M R^{\wedge}(\varrho)$.

3. $\mathcal{L}$ is replete, if and only if $M R^{\wedge}(\mathfrak{L}) \subset M R(\tau, \mathfrak{L})$.

Proof. 1. The proof of 1 is clear.

2. If $\mu \in I R(\sigma, \mathcal{L})$, then $S(\tilde{\mu})=\{\mu\}$, and 2 follows directly.

3. Assume $\mathcal{L}$ is replete, and let $\mu \in M R^{\wedge}(\mathfrak{L})$. Then, $\mu^{\prime} \in M R\left(\tau, W_{\sigma}(\mathcal{L})\right)$, and, by Theorem 2.4, it follows that $\tilde{\mu}(K)=0$ for $K \in t W(\mathcal{L})$ and $K \subset \operatorname{IR}(\mathcal{E})-X$.

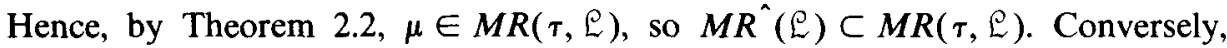
assume $M R^{\wedge}(\mathfrak{L}) \subset M R(\tau, \mathfrak{L})$. If $I R(\sigma, \mathfrak{L}) \neq X$, let $\mu \in I R(\sigma, \mathfrak{L})-X$; then, $\mu \in$ $M R(\tau, \mathcal{L})$, and, by Theorem 2.2 , we get that $\tilde{\mu}(\{\mu\})=0$, a contradiction. Hence, $\operatorname{IR}(\sigma, \mathcal{L})=X$, and $\mathcal{L}$ is replete.

THEOREM 3.2. (On $M R^{-}(\mathcal{Q})$.) The following statements are true:

1. $I R(\sigma, \mathcal{L}) \subset M R^{\tilde{N}}(\mathfrak{L})$.

2. If $\mu \in M R(\mathcal{L})$, then $\mu \in M R^{\tilde{L}}(\mathcal{L})$, if and only if $S(\tilde{\mu}) \subset I R(\sigma, \mathcal{L})$.

3. $\mathcal{L}$ is replete, if and only if whenever $\mu \in M R^{\tilde{L}}(\mathcal{L})$, then $S(\tilde{\mu}) \subset X$.

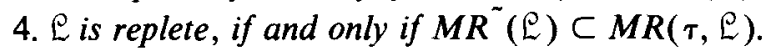

Proof. 1. Let $\mu \in \operatorname{IR}(\sigma, \mathcal{L})$ and $\rho \in \operatorname{IR}(\mathcal{E})-\operatorname{IR}(\sigma, \mathcal{L})$. Then, $\rho \neq \mu$. Hence, there exists a $G \in(t W(\mathscr{L}))^{\prime}$ such that $\rho \in G$ and $\mu \notin G$. Thus, $\tilde{\mu}(G)=0$, and 1 holds.

2. Let $\mu \in M R(\mathcal{L})$ and assume $\mu \in M R^{\tilde{L}}(\mathcal{E})$. If $S(\tilde{\mu}) \not \subset I R(\sigma, \mathcal{E})$, then there exists a $\rho \in I R(\mathcal{L})$ such that $\rho \in S(\tilde{\mu})$ and $\rho \notin I R(\sigma, \mathcal{L})$. But, $\mu \in M R^{-}(\mathcal{L})$, so there exists a $G \in(t W(\mathscr{L}))^{\prime}$ with $\rho \in G$ and $\tilde{\mu}\left(G^{\prime}\right)=\tilde{\mu}(I R(\mathscr{L}))$. Hence, since $\rho \in S(\tilde{\mu}), \rho \in G^{\prime}$, a contradiction. Conversely, assume $S(\tilde{\mu}) \subset I R(\sigma, \mathcal{L})$, and let $\rho \in I R(\mathcal{L})-I R(\sigma, \mathcal{L})$. Then, $\rho \notin S(\tilde{\mu})$; therefore, there exists a $G \in(t W(\mathcal{E}))^{\prime}$ such that $\tilde{\mu}\left(G^{\prime}\right)=\tilde{\mu}(I R(\mathfrak{L}))$ and $\rho \notin G^{\prime}$. Thus, $\rho \in G$ and $\tilde{\mu}(G)=0$, so $\mu \in$ $M R^{\tilde{N}}(\mathfrak{E})$.

3. The proof of 3 is clear.

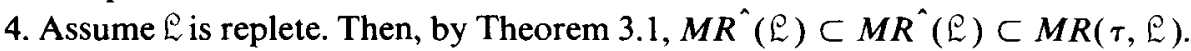
The proof of the converse is not difficult and will be omitted.

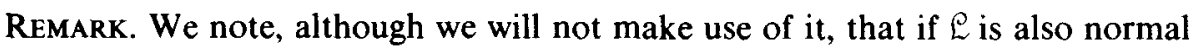

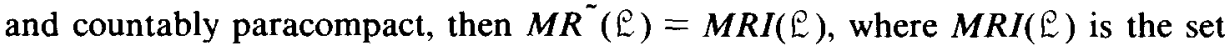
of all $\mu \in M R(\mathcal{L})$ which integrate all $f \in C(\mathcal{E})$. (See [4].) 
LEMMA 3.1. Let $\mathcal{L}$ be any lattice of subsets of $X$ such that $\mathfrak{L}$ is (separating) and disjunctive. If $\lambda \in M R(t W(\mathcal{L}))$ and $\lambda^{*}(X)=\lambda(I R(\mathcal{L}))$, then there exists $a \mu \in$ $M R(\mathcal{Q})$ such that $\lambda=\tilde{\mu}$ and $\mu \in M R(\tau, \varrho)$.

Proof. Consider $\lambda \mid \mathfrak{A}(W(\mathfrak{L}))$, and denote it by $\lambda$ again. Then, there exists a

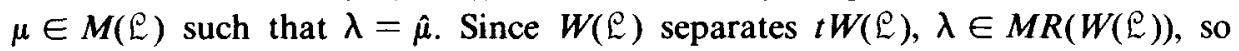
$\mu \in M R(\mathcal{L})$; also, clearly we must have $\lambda=\tilde{\mu}$. Hence, $\tilde{\mu}^{*}(X)=\lambda^{*}(X)=$ $\lambda(I R(\mathcal{L}))=\tilde{\mu}(I R(\mathcal{L}))$, and, by Theorem $2.2, \mu \in M R(\tau, \mathcal{L})$.

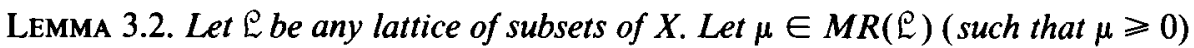
and consider the measures $\hat{\mu}$ on $\sigma(W(\mathcal{E})$ ) and $\tilde{\mu}$ on $\sigma(t W(\varrho))$. (Recall $\hat{\mu}$ is $\delta W(\varrho)$-regular and $\tilde{\mu}$ is $t W(\varrho)$-regalar.) Next, let $H$ be any subset of $I R(\varrho)$. Then,

1. There exists a countably additive measure $\rho$ on $\sigma(W(\mathcal{E}))$ such that $0 \leqslant \rho \leqslant \hat{\mu}, \rho$ is $\delta W(\mathcal{E})$-regular, and $\rho^{*}(H)=\rho(I R(\mathcal{E}))=\hat{\mu}^{*}(H)$.

2. There exists a countably additive measure $\rho$ on $\sigma(t W(\varrho))$ such that $0 \leqslant \rho \leqslant \tilde{\mu}$, $\rho$ is $t W(\mathfrak{E})$-regular, and $\rho^{*}(H)=\rho(\operatorname{IR}(\mathfrak{E}))=\tilde{\mu}^{*}(H)$.

Proof. (For 1.) Since $\hat{\mu}$ is $\delta W(\mathfrak{Q})$-regular, there exists a decreasing sequence $\left\langle A_{n}\right\rangle, A_{n} \in(\delta W(\mathcal{E}))^{\prime}, A_{n} \supset H$ for all $n$, and $\lim _{n} \hat{\mu}\left(A_{n}\right)=\hat{\mu}^{*}(H)$. Then, $A=$ $\cap_{n} A_{n} \in \sigma(W(\varrho))$. Now, define $\rho$ on $\sigma(W(\varrho))$ by $\rho(E)=\hat{\mu}(E \cap A)$, where $E \in \sigma(W(\mathcal{E}))$. Clearly, $\rho$ is a countably additive measure on $\sigma(W(\mathcal{L}))$ and $0 \leqslant \rho \leqslant \hat{\mu}$. Also, since $\hat{\mu}$ is $\delta W(\mathcal{E})$-regular, so is $\rho$. Now, for all $n, \rho^{*}(H) \leqslant \rho\left(A_{n}\right)$ $=\hat{\mu}\left(A_{n} \cap A\right)=\hat{\mu}(A)=\lim _{n} \hat{\mu}\left(A_{n}\right)=\hat{\mu}^{*}(H)$. Hence, $\rho^{*}(H) \leqslant \hat{\mu}(A)=$ $\rho(I R(\mathcal{E})) \leqslant \hat{\mu}^{*}(H)$. But, if $G \in(\delta W(\mathcal{E}))^{\prime}$ and $G \supset H$, then,

$$
\rho(G)=\hat{\mu}(G \cap A)=\lim _{n} \hat{\mu}\left(G \cap A_{n}\right) \geqslant \hat{\mu}^{*}(H),
$$

and the $\delta W(\mathcal{E})$-regularity of $\rho$ implies that $\rho^{*}(H) \geqslant \hat{\mu}^{*}(H)$. Combining this with the above gives $\rho^{*}(H)=\rho(I R(\mathcal{L}))=\hat{\mu}^{*}(H)$.

A similar proof holds for Case 2.

OBSERVATION. $\rho \mid \mathfrak{A}(\delta W(\mathcal{L})) \in M R(\sigma, \delta W(\mathcal{L}))$, and, since $W(\mathcal{E})$ separates

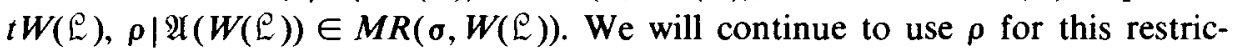
tion.

REMARK. This lemma generalizes a result of Knowles ([10], page 143). 
In this section we define a purely finitely additive measure (p.f.a.), a purely $\sigma$-additive measure (p.o.a), and a purely $\tau$-additive measure (p. $\tau$.a), and for each type of measure we give a characterization theorem.

Let $\mathcal{L}$ be any lattice of subsets of $X$.

Definition 4.1. $\mu \in M R(\varrho)$ is p.f.a. if

$$
\gamma \in M(\sigma, \mathcal{L}) \text { and } 0 \leqslant \gamma \leqslant \mu \text { implies } \gamma=0 \text {. }
$$

THEOREM 4.1. Let $\mathcal{L}$ be any lattice of subsets of $X$ such that $\mathcal{L}$ is (separating) and disjunctive. If $\mu \in M R(\varrho)$, then the following statements are true:

1. If $\mu$ is p.f.a., then $\hat{\mu}^{*}(X)=0$.

2. If $\mathcal{L}$ is $\delta$ and $\sigma(\mathfrak{L}) \subset s(\mathcal{Q})$, and $\hat{\mu}^{*}(X)=0$, then $\mu$ is p.f.a.

Proof. 1. Assume $\mu$ is p.f.a., and $\hat{\mu}^{*}(X) \neq 0$. By Lemma 3.2(1), there exists a $\rho \in M R(\sigma, W(\mathcal{L}))$ such that $0 \leqslant \rho \leqslant \hat{\mu}$ and $\rho^{*}(X)=\rho(I R(\mathscr{L}))=\hat{\mu}^{*}(X)$. Then, $\rho=\hat{\nu}$, where $\nu \in M R(\mathcal{L})$, so $0 \leqslant \hat{\nu} \leqslant \hat{\mu}$, and $0 \leqslant \nu \leqslant \mu$. But, $\hat{\nu}^{*}(X)=\hat{\nu}(\operatorname{IR}(\mathcal{L}))$; therefore, by Theorem 2.1, $\nu \in M R(\sigma, \mathcal{L})$. Thus, since $\mu$ is p.f.a., $\nu=0$; but, $\nu(X)=\hat{\nu}(I R(\mathcal{L}))=\rho(I R(\mathscr{L}))=\hat{\mu}^{*}(X) \neq 0$, a contradiction.

2. Suppose $\mathscr{L}$ is $\delta$ and $\sigma(\mathfrak{L}) \subset s(\mathfrak{L})$, and $\hat{\mu}^{*}(X)=0$. Let $\gamma \in M(\sigma, \mathscr{L})$ and $0 \leqslant \gamma \leqslant \mu$. Then, $0 \leqslant \hat{\gamma} \leqslant \hat{\mu}$, so $0 \leqslant \hat{\gamma}^{*} \leqslant \hat{\mu}^{*}$. Hence, $\hat{\gamma}^{*}(X)=0$. Now, by the assumptions on $\mathcal{L}, \gamma \in M R(\sigma, \mathcal{L})$. Therefore, by Theorem $2.1, \hat{\gamma}^{*}(X)=\hat{\gamma}(\operatorname{IR}(\mathfrak{L}))$, so $\gamma=0$. Hence, $\mu$ is p.f.a.

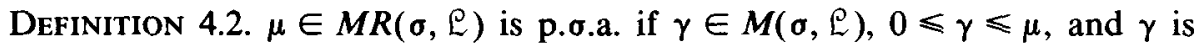
$\tau$-smooth implies $\gamma=0$.

Analogously to Theorem 4.1 we have the following:

THEOREM 4.2. Let $\mathrm{Q}$ be any lattice of subsets of $X$ such that $\mathcal{L}$ is (separating) and disjunctive. If $\mu \in M R(\sigma, \mathcal{L})$, then the following statements are true:

1. If $\mu$ is p.o.a., then $\tilde{\mu}^{*}(X)=0$.

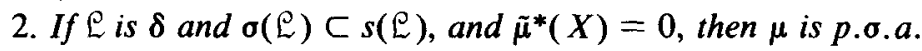

Proof. 1. The proof of this part is quite similar to the proof of the first part of Theorem 4.1. Here, we use Lemma 3.2, Case 2, and Lemma 3.1, and, structurally, the proof is the same as in Theorem 4.1. 
2. The proof of this part is also similar to the proof of the corresponding part of Theorem 4.1, so we will be brief. Let $\gamma \in M(\sigma, \mathcal{L}), 0 \leqslant \gamma \leqslant \mu$, and $\gamma$ be $\tau$-smooth. Then, $0 \leqslant \tilde{\gamma} \leqslant \tilde{\mu}$ on $\mathfrak{A}(W(\mathscr{E}))$. One can then show that $0 \leqslant \tilde{\gamma} \leqslant \tilde{\mu}$ on $\mathfrak{A}(t W(\mathcal{E}))$, by using the fact that $\tilde{\mu} \in M R(\tau, t W(\mathscr{L}))$ and that $\tilde{\gamma} \in M R(\tau, t W(\mathcal{E}))$, since $\mathcal{E}$ is $\delta$ and $\sigma(\mathcal{L}) \subset s(\mathcal{L})$, in conjunction with Lemma 2.2. Then, $0 \leqslant \tilde{\gamma}^{*} \leqslant \tilde{\mu}^{*}$, and, since $\tilde{\mu}^{*}(X)=0$, we get that $\tilde{\gamma}^{*}(X)=0$; but, $\gamma \in M R(\tau, \varrho)$; therefore, by Theorem 2.2, $\tilde{\gamma}(\operatorname{IR}(\mathscr{L}))=0$, and $\gamma=0$, so $\mu$ is p.o.a.

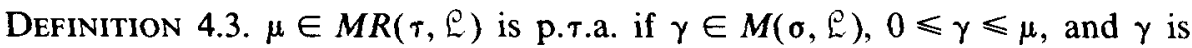
tight implies $\gamma=0$.

THEOREM 4.3. Let $\mathrm{E}$ be any lattice of subsets of $X$ such that $\mathcal{E}$ is separating, disjunctive, and normal. If $\mu \in M R(\tau, \mathcal{L})$, then the following statements are true:

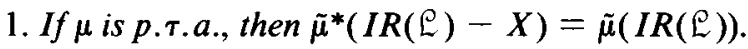

2. If $\mathfrak{E}$ is $\delta$ and $\sigma(\mathfrak{L}) \subset s(\mathfrak{L})$, and $\tilde{\mu}^{*}(\operatorname{IR}(\mathfrak{E})-X)=\tilde{\mu}(\operatorname{IR}(\mathfrak{E}))$, then $\mu$ is p.r.a.

Proof. 1. Suppose $\mu$ is p.t.a. and $\tilde{\mu}^{*}(I R(\mathscr{E})-X)<\tilde{\mu}(\operatorname{IR}(\mathfrak{E}))$. Then, there exists a $G \in(t W(\mathcal{E}))^{\prime}$ such that $G \supset I R(\mathcal{E})-X$ and $\tilde{\mu}(G)<\tilde{\mu}(I R(\mathcal{E}))$. Thus, $I R(\mathscr{Q})-G \in t W(\mathfrak{Q})$. Let $F=I R(\mathfrak{E})-G$. Then, $F$ is $t W(\mathfrak{Q})$-compact and $F \subset X$.

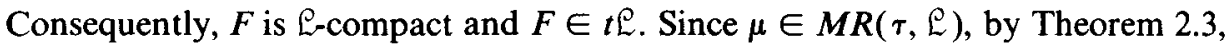

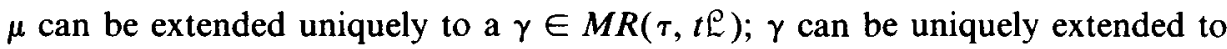
$\sigma(t \mathscr{L})$ and is $t \mathcal{L}$-regular there also. Now, define $\lambda$ on $\sigma(\mathcal{L})$ by $\lambda(E)=\gamma(E \cap F)$. Clearly, $\lambda \in M(\sigma, \mathcal{L})$ and $0 \leqslant \lambda \leqslant \mu$. Let $\varepsilon>0$. Since $F \in t \mathscr{L}, F=\cap_{\alpha} L_{\alpha}$, $L_{\alpha} \in \mathcal{L}$. Using Lemma 2.2 , it is not difficult to see that $\lambda^{*}\left(\cap_{\alpha} L_{\alpha}\right)=$ $\gamma\left(\left(\cap_{\alpha} L_{\alpha}\right) \cap F\right)$. But, $\gamma\left(\left(\cap_{\alpha} L_{\alpha}\right) \cap F\right)=\gamma(F)=\lambda(X)>\lambda(X)-\varepsilon$, so $\lambda$ is tight. Thus, $\lambda=0$; however, $\lambda(X)=\gamma(F)=\tilde{\mu}(F)=\tilde{\mu}(I R(\mathcal{L}))-\tilde{\mu}(G)>0$, a contradiction.

2. Suppose $\mathcal{E}$ is $\delta$ and $\sigma(\mathfrak{L}) \subset s(\mathfrak{L})$, and $\tilde{\mu}^{*}(\operatorname{IR}(\mathfrak{L})-X)=\tilde{\mu}(\operatorname{IR}(\mathfrak{Q}))$. Let $\gamma \in M(\sigma, \mathcal{L}), 0 \leqslant \gamma \leqslant \mu$, and $\gamma$ be tight. Then, $\gamma \in M R(\sigma, \mathcal{E}), 0 \leqslant \tilde{\gamma} \leqslant \tilde{\mu}$ on $\mathfrak{A}(t W(\mathfrak{E}))$, and, by regularity, $0 \leqslant \tilde{\gamma} \leqslant \tilde{\mu}$ on $\sigma(t W(\mathcal{E}))$. Hence, $0 \leqslant \tilde{\gamma}_{*}(X) \leqslant$ $\tilde{\mu}_{*}(X)$, so $\tilde{\gamma}_{*}(X)=0$. Now, $\gamma \in M R(t, \mathcal{L})$, and using Theorem 2.5 , we get that also $\tilde{\gamma}^{*}(X)=0$, so $\tilde{\gamma}(\operatorname{IR}(\mathfrak{L}))=0, \gamma=0$, and $\mu$ is p.r.a.

In this section we define a strongly $\sigma$-additive measure (s.o.a.) and a strongly $\tau$-additive measure (s. $\tau$.a.), and, for each of these types, we also give a characterization theorem. 
Again, let $\mathcal{E}$ be any lattice of subsets of $X$.

Definition 5.1. $\mu \in M R(\sigma, \mathfrak{L})$ is s.o.a. if for $\gamma \in M(\mathfrak{L}), 0 \leqslant \gamma \leqslant \mu, \gamma^{\prime} \in$ $M\left(\sigma, W_{0}(\varrho)\right)$, and $\gamma^{\prime}$ is $\tau$-smooth implies $\gamma=0$.

LEMMA 5.1. Let $\mathcal{E}$ be any lattice of subsets of $X$ such that $\mathcal{L}$ is disjunctive. If $\lambda \in M R(t W(\mathscr{L}))$ and $\lambda^{*}(I R(\sigma, \mathfrak{L}))=\lambda(\operatorname{IR}(\mathfrak{L}))$, then there exists an element $\mu$ of $M R(\mathcal{E})$ such that $\lambda=\tilde{\mu}$ and $\mu^{\prime} \in M R\left(\tau, W_{\sigma}(\mathfrak{L})\right.$ ) (that is, $\mu \in M R^{\wedge}(\mathfrak{L})$ ).

Proof. Consider $\lambda \mid \mathfrak{A}(W(\mathcal{E}))$, and denote it by the same symbol. Then, $\lambda=\hat{\mu}$, where $\mu \in M R(\varrho)$, and $\lambda=\tilde{\mu}$ (see Lemma 3.1); hence, $\tilde{\mu}^{*}(I R(\sigma, \varrho))=\tilde{\mu}(\operatorname{IR}(\varrho))$, and, by Theorem $2.4, \mu^{\prime} \in M R\left(\tau, W_{\sigma}(\varrho)\right)$.

REMARK. $\mu$ need not belong to $M R(\tau, \mathfrak{L})$. For this to be true, $\tilde{\mu}^{*}(X)=\tilde{\mu}(I R(\mathcal{L}))$ must hold.

THEOREM 5.1. Let $\mathcal{E}$ be any lattice of subsets of $X$ such that $\mathcal{E}$ is disjunctive. If $\mu \in M R(\sigma, \mathcal{E})$, then the following statements are true:

1. If $\mu$ is s.o.a., then $\tilde{\mu}^{*}(I R(\sigma, \mathscr{L}))=0$.

2. If $W_{\sigma}(\mathfrak{E})$ is $\delta$ and $\sigma\left(W_{\sigma}(\mathfrak{E})\right) \subset s\left(W_{\sigma}(\mathcal{L})\right)$, and $\tilde{\mu}^{*}(\operatorname{IR}(\sigma, \mathfrak{L}))=0$, then $\mu$ is s.o.a.

OUTLINE OF PROOF. 1. Suppose $\mu$ is s.o.a. and $\tilde{\mu}^{*}(\operatorname{IR}(\sigma, \mathfrak{L})) \neq 0$. By Lemma 3.2, Case 2, there exists a $\rho \in M R(\sigma, t W(\mathcal{L}))$ such that $0 \leqslant \rho \leqslant \tilde{\mu}$ and $\rho^{*}(\operatorname{IR}(\sigma, \mathcal{L}))=\rho(\operatorname{IR}(\mathcal{L}))=\tilde{\mu}^{*}(\operatorname{IR}(\sigma, \mathcal{L}))$. Denote $\rho \mid \mathfrak{A}(W(\mathcal{L}))$ by $\rho$ again. Then, $\rho=\hat{\nu}$, where $\nu \in M(\mathcal{L})$, and $0 \leqslant \hat{\nu} \leqslant \hat{\mu}$, so $0 \leqslant \nu \leqslant \mu$. By Lemma $5.1, \rho=\tilde{\nu}$ and $\nu^{\prime} \in M R\left(\tau, W_{0}(\mathcal{L})\right)$. Thus, $\quad \nu=0 ; \quad$ but, $\quad \nu(X)=\tilde{\nu}(\operatorname{IR}(\mathfrak{L}))=\rho(\operatorname{IR}(\mathcal{E}))=$ $\tilde{\mu}^{*}(\operatorname{IR}(\sigma, \mathcal{L})) \neq 0$, a contradiction.

2. Just imitate the proof of the corresponding part of Theorem 4.2 using Theorem 2.4 instead of Theorem 2.2.

Remark. Note that the condition $\sigma\left(W_{\sigma}(\mathfrak{E})\right) \subset s\left(W_{\sigma}(\mathfrak{E})\right)$ is satisfied, for example, when $\sigma\left(W_{o}(\mathscr{L})\right)=\rho\left(W_{o}(\mathcal{L})\right)$. Hence, in particular, when $\mathscr{L}$ is complement generated, in which case, $W_{\sigma}(\mathcal{L})$ is complement generated.

EXAMPLE. Let $X$ be a $T_{3 \frac{1}{2}}$ topological space and $\mathcal{L}=\mathscr{Z}$, the lattice of zero sets. In this case, $I R(\sigma, \mathscr{Z})=v X$, the real compactification of $X$. 
Observation 1. Let $e$ be a disjunctive lattice of subsets of $X$. Let $\rho \in$ $M\left(\sigma, W_{\sigma}(\mathcal{E})\right)$. Then, $\rho=\nu^{\prime}$, where $\nu \in M(\sigma, \mathfrak{L})$, and, consequently, the following is true: If $\mathscr{L}$ is $\delta$ and $\sigma(\mathscr{L}) \subset s(\mathscr{L})$, and $\tilde{\mu}^{*}(\operatorname{IR}(\sigma, \mathscr{L}))=0$, then $\mu$ is s.o.a. (The details will be omitted.)

OBSERVATION 2. If $\mathscr{L}$ is (separating), disjunctive, $\delta$ and $\sigma(\mathscr{L}) \subset s(\mathfrak{L})$, then $\mu$ is s.o.a. implies $\mu$ is p.o.a., for, by Theorem 5.1, Part $1, \tilde{\mu}^{*}(\operatorname{IR}(\sigma, \mathcal{L}))=0$. Hence, $\tilde{\mu}^{*}(X)=0$, and, by Theorem 4.2, Part $2, \mu$ is p.o.a.

Definition 5.2. $\mu \in M R(\tau, \mathfrak{L})$ is s.t.a. if for $\gamma \in M(\mathfrak{L}), 0 \leqslant \gamma \leqslant \mu, \gamma^{\prime} \in$ $M\left(\sigma, W_{\sigma}(\mathfrak{L})\right)$, and $\gamma^{\prime}$ is tight implies $\gamma=0$.

By imitating the proof of Theorem 2.5, we can establish the following:

Lemma 5.2. Let $\mathfrak{L}$ be any lattice of subsets of $X$ such that $\mathfrak{L}$ is separating, disjunctive, and normal. If $\mu \in M R(\varrho)$, then the following statements are equivalent:

1. $\mu^{\prime} \in M R\left(t, W_{0}(\mathcal{L})\right)$.

2. $\tilde{\mu}^{*}(\operatorname{IR}(\sigma, \varrho))=\tilde{\mu}(I R(\varrho))$ and $\operatorname{IR}(\sigma, \varrho)$ is $\tilde{\mu}^{*}$-measurable.

CoROllaRY 1. If $\mathcal{E}$ is also replete and $\mu \in M R(\mathfrak{E})$, then $\mu^{\prime} \in M R\left(t, W_{\sigma}(\mathfrak{E})\right)$, if and only if $\mu \in M R(t, \mathcal{E})$.

Proof. Use Lemma 5.2 and Theorem 2.5.

Note, if $\mathcal{L}$ is disjunctive and replete and $\mu \in M R(\mathcal{E})$, then $\mu \in M R^{\wedge}(\mathscr{L})$, if and only if $\mu^{\prime} \in M R\left(\tau, W_{o}(\mathcal{L})\right)$, if and only if $\mu \in M R(\tau, \mathcal{L})$.

Corollary 2. If $\mu \in M R(\mathfrak{L})$, then $\mu \in M R(t, \mathfrak{L})$ implies $\mu^{\prime} \in M R\left(t, W_{o}(\mathfrak{L})\right)$.

Proof. By Theorem 2.5, $\tilde{\mu}^{*}(X)=\tilde{\mu}(I R(\mathcal{L}))$ and $X$ is $\tilde{\mu}^{*}$-measurable. Clearly, then $\tilde{\mu}^{*}(I R(\sigma, \mathcal{L}))=\tilde{\mu}(\operatorname{IR}(\mathcal{L}))$. Hence, $\tilde{\mu}_{*}(X) \leqslant \tilde{\mu}_{*}(I R(\sigma, \mathcal{L})) \leqslant \tilde{\mu}^{*}(\operatorname{IR}(\sigma, \mathcal{L})) \leqslant$ $\tilde{\mu}^{*}(X)$, so $I R(\sigma, \mathcal{L})$ is $\tilde{\mu}^{*}$-measurable, and, by Lemma 5.2, $\mu^{\prime} \in M R\left(t, W_{\sigma}(\mathcal{E})\right)$.

Corollary 3. Let $\mu \in M R(\mathcal{E})$. Then, $\mu^{\prime} \in M R\left(t, W_{\sigma}(\mathfrak{Q})\right)$, if and only if there exists a $G_{\delta}$-set, $H$, of $I R(\varrho)$ such that $H \supset I R(\mathscr{E})-I R(\sigma, \varrho)$ and $\tilde{\mu}(H)=0$.

Proof. Just use Lemma 5.2 and the $t W(\mathfrak{L})$-regularity of $\tilde{\mu}$. 
Note, if every $G_{\delta}$-set of $\operatorname{IR}(\mathfrak{L})$ is open, then $\mu^{\prime} \in M R\left(t, W_{\sigma}(\mathfrak{L})\right)$ implies $\mu \in M R^{\sim}(e)$.

Finally, by imitating the proof of Theorem 4.3, we can establish the following:

THEOREM 5.2. Let $\mathfrak{L}$ be any lattice of subsets of $X$ such that $\mathcal{L}$ is separating, disjunctive, and normal. If $\mu \in M R(\tau, \mathcal{L})$, then the following statements are true:

1. If $\mu$ is s.T.a., then $\tilde{\mu}^{*}(\operatorname{IR}(\mathfrak{L})-\operatorname{IR}(\sigma, \mathfrak{L}))=\tilde{\mu}(\operatorname{IR}(\mathfrak{L}))$.

2. If $W_{\mathrm{o}}(\mathfrak{E})$ is $\delta$ and $\sigma\left(W_{\mathrm{o}}(\mathfrak{L})\right) \subset s\left(W_{\mathrm{o}}(\mathfrak{E})\right)$, and $\tilde{\mu}^{*}(\operatorname{IR}(\mathfrak{Q})-\operatorname{IR}(\sigma, \mathfrak{E}))=$ $\tilde{\mu}(I R(\mathfrak{L}))$, then $\mu$ is s.t.a.

\section{6}

In this section we give a characterization of repleteness in terms of p.o.a. and s.o.a. measures; we also present a relationship between p.o.a. and s.o.a. measures, under repleteness, and a relationship between p. $\tau$.a. measures, under repleteness.

THEOREM 6.1. Let $\mathcal{E}$ be any lattice of subsets of $X$ such that $\mathcal{E}$ is (separating), disjunctive, $\delta$ and $\sigma(\mathcal{E}) \subset s(\mathfrak{L})$. Then, $\mathcal{E}$ is replete, if and only if for any $\mu \in$ $\operatorname{MR}(\sigma, \mathcal{E}), \mu$ is p.o.a. implies $\mu$ is s.o.a.

Proof. $\alpha)$. Suppose $E$ is replete, and $\mu \in M R(\sigma, E)$ is p.o.a. Then, by Theorem 4.2. Part $1, \tilde{\mu}^{*}(X)=0$. But, $I R(\sigma, \mathcal{L})=X$; therefore, $\tilde{\mu}^{*}(I R(\sigma, \mathcal{L}))=0$. Also, $W_{o}(\mathfrak{E})=\mathfrak{E}$, and, by Theorem 5.1, Part 2, $\mu$ is s.o.a.

$\beta)$. Assume the condition holds. If $\operatorname{IR}(\sigma, \mathcal{L}) \neq X$, let $\mu \in \operatorname{IR}(\sigma, \mathcal{E})-X$. Then, $S(\tilde{\mu})=\{\mu\}$, and, since $\tilde{\mu}$ is $\tau W(\mathcal{L})$-regular, it follows that $\tilde{\mu}^{*}(X)=0$, and, Theorem 4.2, Part 2, implies that $\mu$ is p.o.a. Hence, by the assumption, $\mu$ is s.o.a. Thus, by Theorem 5.1, Part 1, $\tilde{\mu}^{*}(\operatorname{IR}(\sigma, \mathcal{E}))=0$, a contradiction since $\tilde{\mu}(\{\mu\})=1$.

Application. Let $X$ be a $T_{3 \frac{1}{2}}$ topological space and $\mathcal{L}=\mathscr{Z}$, the lattice of zero sets. Then, by the preceding theorem, $\mathscr{L}$ is replete, that is, $X$ is realcompact, if and only if, for every $\mu \in M R(\sigma, \mathscr{Z}), \mu$ is p.o.a. implies $\mu$ is s.o.a.

THEOREM 6.2. Let $\mathcal{L}$ be any lattice of subsets of $X$ such that $\mathcal{L}$ is separating, disjunctive, normal, $\delta$ and $\sigma(\mathfrak{L}) \subset s(\mathfrak{L})$. The following statement is true: If $\mu$ is s.T.a., then $\mu$ is p.T.a.

Proof. By Theorem 5.2, Part 1, $\tilde{\mu}^{*}(\operatorname{IR}(\mathcal{L})-\operatorname{IR}(\sigma, \mathcal{E}))=\tilde{\mu}(\operatorname{IR}(\mathscr{E}))$; hence, $\tilde{\mu}^{*}(I R(\varrho)-X)=\tilde{\mu}(I R(巳))$, and, using Theorem 4.3, Part 2, completes the proof. 
Observation. Assume that $\mathfrak{L}$ is also replete. Then, for $\mu \in M R(\tau, \mathfrak{L}), \mu$ is s. $\tau$.a., if and only if $\mu$ is p. $\tau$.a. (Proof omitted.)

In this section, as a result of our previous development, we extend the well-known Yosida-Hewitt Decomposition Theorem go more general lattices than the complemented ones (that is, algebras) and we even obtain further refinements.

THEOREM 7.1. Let $\mathcal{Q}$ be any lattice of subsets of $X$ such that $\mathcal{E}$ is separating, disjunctive, normal, $\delta$ and $\sigma(\mathfrak{L}) \subset s(\mathfrak{L})$. The following statement is true: if $\mu \in$

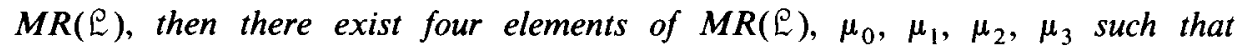
$\mu=\mu_{0}+\mu_{1}+\mu_{2}+\mu_{3}$, and $\mu_{0}$ is p.f.a., $\mu_{1}$ is p.o.a., $\mu_{2}$ is p.t.a., and $\mu_{3}$ is tight; moreover, such a representation of $\mu$ is unique.

Proof. Existence. $\alpha$ ). Consider $\hat{\mu}$. By Lemma 3.2, Case 1, there exists a $\rho \in M R(\sigma, W(\mathcal{L}))$ such that $0 \leqslant \rho \leqslant \hat{\mu}$ and $\rho^{*}(X)=\rho(I R(\mathcal{L}))=\hat{\mu}^{*}(X)$. Now, $\rho=\hat{\nu}$, where $\nu \in M R(\mathcal{L})$. Then, $0 \leqslant \hat{\nu} \leqslant \hat{\mu}$, so $0 \leqslant \nu \leqslant \mu$. Also, $\hat{\nu}^{*}(X)=$ $\hat{\nu}(I R(\mathcal{E}))$; hence, $\nu \in M R(\sigma, \mathcal{L})$. Now, let $\mu_{0}=\mu-\nu$; then, $\mu_{0} \geqslant 0$ and $\mu_{0} \in$ $\operatorname{MR}(\mathfrak{L})$. Since $\mu=\mu_{0}+\nu, \hat{\mu}=\hat{\mu}_{0}+\hat{\nu}$. Thus, $\hat{\mu}^{*}=\hat{\mu}_{0}^{*}+\hat{\nu}^{*}$ (see [19], page 33). Consequently, $\hat{\mu}_{0}^{*}(X)=\hat{\mu}^{*}(X)-\hat{\nu}^{*}(X)=0$, and, by Theorem 4.1, Part 2, $\mu_{0}$ is p.f.a. Hence, $\mu=\mu_{0}+\nu$, where $\mu_{0}$ is p.f.a. and $\nu \in M R(\sigma, \mathcal{L})$.

$\beta)$. Next, consider $\tilde{\boldsymbol{\nu}}$. By Lemma 3.2, Case 2, there exists a $\lambda \in M R(t W(\mathfrak{L}))$ such that $0 \leqslant \lambda \leqslant \tilde{\nu}$ and $\lambda^{*}(X)=\lambda(I R(\mathcal{L}))=\tilde{\nu}^{*}(X)$. Then, $\lambda=\tilde{\tau}$, where $\tau \in$ $M R(\tau, \mathcal{L})$, by Lemma 3.1. It follows that $0 \leqslant \hat{\tau} \leqslant \hat{\nu}$, so $0 \leqslant \tau \leqslant \nu$. Now, let $\mu_{1}=\nu-\tau ;$ then, $\mu_{1} \geqslant 0$ and $\mu_{1} \in M R(\sigma, \mathcal{E})$. Since $\nu=\mu_{1}+\tau, \tilde{\nu}=\tilde{\mu}_{1}+\tilde{\tau}$, and $\tilde{\nu}^{*}=\tilde{\mu}_{1}^{*}+\tilde{\tau}^{*}$, and it follows that $\tilde{\mu}_{1}^{*}(X)=0$. Part 2 of Theorem 4.2 implies that $\mu_{1}$ is p.o.a. Consequently, $\mu=\mu_{0}+\mu_{1}+\tau$, where $\mu_{0}$ is p.f.a., $\mu_{1}$ is p.o.a., and

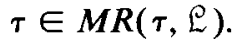

$\gamma)$. Next, consider $\tilde{\tau}$. Since $\tilde{\tau}$ is $t W(\varrho)$-regular, there exists a sequence $\left\langle K_{n}\right\rangle$ in $t W(\varrho)$ such that $K_{n} \subset X$ for all $n,\left\langle K_{n}\right\rangle$ is increasing, and $\lim _{n} \tilde{\tau}\left(K_{n}\right)=\tilde{\tau}_{*}(X)$. Let $H=\cup_{n} K_{n} \in \sigma(t W(\mathfrak{L}))$. Then, $\tilde{\tau}(H)=\tilde{\tau}_{*}(X)$. Now, define $\omega$ on $\sigma(t W(\mathfrak{L}))$ by $\omega(E)=\tilde{\tau}(E-H)$. Consider $\omega \mid \mathfrak{A}(t W(\mathfrak{L}))$, and denote it by the same symbol; clearly, $\omega \in M R(t W(\mathfrak{L}))$.

Also, if $K \in t W(\mathfrak{Q}), K \subset I R(\mathfrak{Q})-X$, then $\omega(K)=\tilde{\tau}(K-H) \leqslant \tilde{\tau}(K)=0$, since $\tau \in M R(\tau, \mathscr{E})$ (see Theorem 2.2). Hence, $\omega_{*}(I R(\mathscr{E})-X)=0$, so $\omega^{*}(X)=$ $\omega(\operatorname{IR}(\mathcal{Q}))$. Then, Lemma 3.1 implies that $\omega=\tilde{\mu}_{2}$, where $\mu_{2} \in M R(\tau, \mathfrak{E})$. Also, 
since $\omega$ is $t W(\mathfrak{L})$-regular, it is easy to see that $\omega_{*}(X)=0$, so $\omega^{*}(I R(\mathcal{L})-X)=$ $\omega(\operatorname{IR}(\mathfrak{E}))$. Therefore, $\tilde{\mu}_{2}^{*}(\operatorname{IR}(\mathfrak{L})-X)=\tilde{\mu}_{2}(\operatorname{IR}(\mathfrak{E}))$, and Theorem 4.3, Part 2, implies that $\mu_{2}$ is p. $\tau$.a.

$\delta)$. Define $\varepsilon$ on $\mathfrak{A}(\mathcal{Q})$ by $\varepsilon(E)=\tilde{\tau}(W(E) \cap H)$. Let $\left\langle E_{n}\right\rangle$ be any decreasing sequence in $\mathfrak{A}(\mathcal{L})$ such that $\lim _{n} E_{n}=\varnothing$. Then, $\cap_{n} W\left(E_{n}\right) \subset \operatorname{IR}(\mathfrak{E})-X$, and $\lim _{n} \varepsilon\left(E_{n}\right)=\lim _{n} \tilde{\tau}\left(W\left(E_{n}\right) \cap H\right)=\tilde{\tau}\left(\cap_{n}\left(W\left(E_{n}\right) \cap H\right)\right) \leqslant \tilde{\tau}\left(\cap_{n} W\left(E_{n}\right)\right)$. If $\tilde{\tau}\left(\cap_{n} W\left(E_{n}\right)\right)>0$, then, since $\tilde{\tau}$ is $t W(\mathfrak{L})$-regular, there exists a $K \in t W(\mathfrak{E})$ such that $K \subset \cap_{n} W\left(E_{n}\right)$ and $\tilde{\tau}(K)>0$. But, $\tau \in M R(\tau, \mathcal{L})$, and, therefore, we have a contradiction (see Theorem 2.2). Thus, $\varepsilon \in M(\sigma, \mathcal{L})$, and, since $\mathcal{L}$ is $\delta$ and

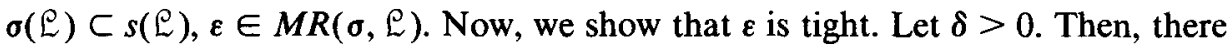
exists a $K \in t W(\mathscr{L})$ such that $K \subset X$ and $\tilde{\tau}(K)>\tilde{\tau}_{*}(X)-\delta . K$ is, of course, $t W(\mathfrak{L})$-compact. Hence, since $K \subset X, K$ is -compact. Also,

$\varepsilon_{*}\left(K^{\prime}\right)=\varepsilon_{*}(X-K)=\sup \left\{\varepsilon(L) \mid L \in \rho_{\infty} L \subset X-K\right\}$

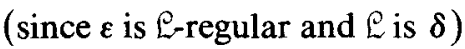

$$
\begin{aligned}
& =\sup \{\tilde{\tau}(W(L) \cap H) \mid L \in \mathcal{L}, L \subset X-K\} \\
& \leqslant \sup \{\tilde{\tau}(W(L) \cap X) \mid L \in \mathcal{L}, W(L) \cap X \subset X-K\} \\
& \leqslant \tilde{\tau}_{*}(X-K) .
\end{aligned}
$$

However, $\tilde{\tau}_{*}(X) \geqslant \tilde{\tau}_{*}(K)+\tilde{\tau}_{*}(X-K)=\tilde{\tau}(K)+\tilde{\tau}_{*}(X-K)$. Thus, $\tilde{\tau}_{*}(X-K)<\delta$. Consequently, $\varepsilon$ is tight. Let $\mu_{3}=\varepsilon$.

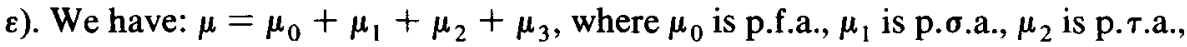
and $\mu_{3}$ is ( $(-$-regular) and tight.

Uniqueness. Consider any two representations of $\mu$ of the type obtained above, namely, $\mu=\mu_{0}+\mu_{1}+\mu_{2}+\mu_{3}$ and $\mu=\nu_{0}+\nu_{1}+\nu_{2}+\nu_{3}$, with $\mu_{0}$ and $\nu_{0}$ p.f.a., $\mu_{1}$ and $\nu_{1}$ p.o.a., $\mu_{2}$ and $\nu_{2}$ p.r.a., and $\mu_{3}$ and $\nu_{3}$ (L-regular) and tight. Denote $\mu_{1}+\mu_{2}+\mu_{3}$ by $\lambda$ and $\nu_{1}+\nu_{2}+\nu_{3}$ by $\rho$. Then, $\mu_{0}+\lambda=\nu_{0}+\rho$, and $\mu_{0}$ and $\nu_{0}$ are p.f.a., and $\lambda \in M R(\sigma, \mathcal{L})$ and $\rho \in M R(\sigma, \mathcal{L})$. Then, $\lambda-\rho=\nu_{0}-\mu_{0}$. Hence, since $\mu_{0} \geqslant 0, \lambda-\rho \leqslant \nu_{0}$. Also, since $\nu_{0} \geqslant 0,0 \leqslant(\lambda-\rho)^{+} \leqslant \nu_{0}$ and $0 \leqslant$ $-(\lambda-\rho)^{-} \leqslant \nu_{0}$. Thus, since $(\lambda-\rho)^{+} \in M(\sigma, \mathcal{L})$ and $-(\lambda-\rho)^{-} \in M(\sigma, \ell)$, and $\nu_{0}$ is p.f.a., $(\lambda-\rho)^{+}=0$ and $-(\lambda-\rho)^{-}=0$. Therefore, $\lambda-\rho=0$. Consequently, $\mu_{0}=\nu_{0}$ and $\mu_{1}+\mu_{2}+\mu_{3}=\nu_{1}+\nu_{2}+\nu_{3}$.

One can proceed similarly to show $\mu_{1}=\nu_{1}$, then $\mu_{2}=\nu_{2}$, and then $\mu_{3}=\nu_{3}$.

Remark. The decomposition theorem just proved is applicable, for example, to any $T_{3 \frac{1}{2}}$ space, with $\mathcal{L}=\mathscr{I}$, or to any normal analytic space, with $\mathcal{L}$ the collection of its closed sets. 


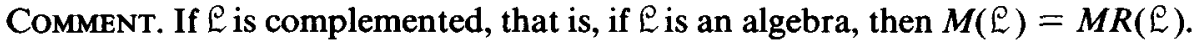
Hence, in this case, we can dispense with the condition $\mathcal{L}$ is $\delta$ and $\sigma(\mathcal{L}) \subset s(\mathcal{L})$. Also, in this case, $\mathcal{E}$ is disjunctive, and since in the first part of the decomposition theorem it is not required that $E$ be either separating or normal, we obtain the following:

Corollary. If $\mu \in M(\mathfrak{Q})$, then there exist $\mu_{0}, \nu \in M(\mathfrak{L})$ such that $\mu=\mu_{0}+\nu$, and $\mu_{0}$ is p.f.a. and $\nu \in M(\sigma, \varrho)$; moreover, such a representation of $\mu$ is unique.

This special case is the well-known Yosida-Hewitt Decomposition Theorem, [18].

Observation. Let $\mu \in M R(\sigma, \mathcal{L})$ be p.o.a. By Theorem 4.2(1.), $\tilde{\mu}^{*}(X)=0$. By Lemma 3.2, Case 2, there exists a $\rho \in M R(t W(\mathscr{L}))$ such that $0 \leqslant \rho \leqslant \tilde{\mu}$ and $\rho^{*}(\operatorname{IR}(\sigma, \mathfrak{E}))=\rho(\operatorname{IR}(\mathfrak{E}))=\tilde{\mu}^{*}(I R(\sigma, \mathcal{L}))$. Consider $\rho / \mathfrak{A}(W(\mathfrak{L}))$, and denote it by the same symbol. Then, $\rho=\hat{\nu}$, where $\nu \in M(\varrho)$. Clearly, $0 \leqslant \hat{\nu} \leqslant \hat{\mu}$, so $0 \leqslant \nu \leqslant \mu$. By Lemma 5.1, $\rho=\tilde{\nu}$ and $\nu^{\prime} \in M R\left(\tau, W_{\mathrm{o}}(\mathfrak{E})\right)$. Let $\lambda=\mu-\nu$. Then, $\lambda \geqslant 0$ and, since $\mu, \nu \in M R(\sigma, \mathcal{L}), \lambda \in M R(\sigma, \mathcal{L})$. Clearly, $\tilde{\mu}^{*}=\tilde{\lambda}^{*}+\tilde{\nu}^{*}$, and it follows that $\tilde{\lambda}^{*}(I R(\sigma, \mathcal{L}))=0$. Hence, since $\mathcal{L}$ is $\delta$ and $\sigma(\mathcal{L}) \subset s(\mathfrak{L})$, by Theorem 5.1 , Observation $1, \lambda$ is s.o.a. However, since $0 \leqslant \tilde{\nu} \leqslant \tilde{\mu}$, we get that $0 \leqslant \tilde{\nu}^{*}(X) \leqslant$ $\tilde{\mu}^{*}(X)=0$, and, by Theorem 4.2, Part 2, $\nu$ is p.o.a. Consequently, $\mu=\lambda+\nu$, where $\lambda$ is s.o.a. and $\nu$ is p.o.a.

One can proceed similarly to obtain a further decomposition of the general p. $\tau$.a. measure and hence a further refinement of the decomposition obtained in the theorem.

\section{Acknowledgement}

The authors wish to express their thanks and appreciation to the referee for his many helpful comments and useful suggestions in the revision of the paper.

\section{References}

[1] A. D. Alexandroff, 'Additive set functions in abstract spaces', Mat. Sb. (N.S.) 9, 51 (1941), 563-628.

[2] G. Bachman and A. Sultan, 'Regular lattice measures: mappings and spaces', Pacific J. Math. 67 (1976), 291-321.

[3] G. Bachman and A. Sultan, 'Extensions of regular lattice measures with topological applications', J. Math. Anal. Appl. 57 (1977), 539-559. 
[4] G. Bachman and A. Sultan, 'Representations of linear functionals on spaces of continuous functions, repletions, and general measure extensions', J. Math. Anal. Appl. 67 (1979), 277-293.

[5] G. Bachman and A. Sultan, 'On regular extensions of measures', Pacific J. Math., to appear.

[6] B. Banaschewski, 'Über nulldimensionalle Raume', Math. Nachr. 13 (1955), 129-140.

[7] L. Gillman and M. Jerrison, Rings of continuous functions (Van Nostrand, Princeton, N.J., (1960)).

[8] G. G. Gould and M. Mahowald, 'Measures on completely regular spaces', J. London Math. Soc. 37 (1962), 103-111.

[9] P. Halmos, Measure theory (Van Nostrand, Toronto, New York, London, (1950)).

[10] J. Knowles, 'Measures on topological spaces', Proc. London Math. Soc. 17 (1967), 139-156.

[11] J. Lembcke, 'Konservative Abbildungen und Fortsetzung regulärer Masse', Z. Wahrscheinlichkeitstheorie und Verw. Gebiete 15 (1970), 57-96.

[12] W. Moran, 'Measures and mappings on topological spaces', Proc. London Math. Soc. (3) 19 (1969), 493-508.

[13] A. Sultan, 'Measure compactification and representation', Canad. J. Math. 30 (1978), 54-65.

[14] M. Szeto, 'Measure repleteness and mapping preservations', J. Indian Math. Soc., to appear.

[15] F. Topsee, Topology and measure, Springer Lecture Notes 133 (Springer-Verlag, Berlin, Heidelberg, New York, (1970)).

[16] V. Varadarajan, 'Measures on topological spaces', Amer. Math. Soc. Transl. Ser. 2. 48 (1965), 161-228.

[17] H. Wallman, 'Lattices and topological spaces', Ann. of Math. 42 (1938), 687-697.

[18] K. Yosida and E. Hewitt, 'Finitely additive measures', Trans. Amer. Math. Soc. 72 (1952), 46-66.

[19] A. Zaanen, An introduction to the theory of integration (North-Holland Publishing Co., Amsterdam, New York, (1958)).

Department of Mathematics

Polytechnic Institute of New York

Brooklyn, New York 11201

U.S.A.
Department of Mathematics

Long Island University

Brooklyn, New York 11201

U.S.A. 